
Nanobeads for High-Efficiency

SUBJECT AREAS:

SOLAR CELLS

ELECTRONIC MATERIALS

NANOPARTICLES

ELECTRONIC DEVICES

Received

21 August 2014

Accepted

14 November 2014

Published

8 December 2014

Correspondence and requests for materials should be addressed to S.Y.J. (syjang@ kookmin.ac.kr) or D.K. (dongho@yonsei.ac. $\mathrm{kr})$ Dye-Sensitized Solar Cells

\author{
Daesub Hwang 1 ,3, Jun-Su Jin ${ }^{2}$, Horim Lee ${ }^{3}$, Hae-Jin Kim ${ }^{4}$, Heejae Chung ${ }^{1}$, Dong Young Kim³, \\ Sung-Yeon Jang ${ }^{2} \&$ Dongho Kim ${ }^{1}$
}

\begin{abstract}
${ }^{1}$ Department of Chemistry, Yonsei University, Seoul 120-749, Korea, ${ }^{2}$ Department of Chemistry, Kookmin University, Seoul $136-$ 702, Korea, ${ }^{3}$ Optoelectronic Materials Lab, Korea Institute of Science and Technology, Seoul 136-791, Korea, ${ }^{4}$ Department of Mechanical Engineering, Yonsei University, Seoul 120-749, Korea.
\end{abstract}

We developed a unique strategy for fabricating hierarchically structured (nanoparticles-in-beads) $\mathrm{Zn}_{2} \mathrm{SnO}_{4}$ beads (ZTO-Bs), which were then used to produce ternary metal oxide-based dye-sensitized solar cells (DSSCs). DSSCs were fabricated using the ZTO-Bs as the photoelectrodes and highly absorbable organic dyes as the sensitizers. The DSSCs based on the ZTO-Bs and the organic dyes (SJ-E1 and SJ-ET1) exhibited the highest performance ever reported for DSSCs with ternary metal oxide-based photoelectrodes. The optimized DSSCs exhibited a power conversion efficiency of $6.3 \%\left(\mathrm{~V}_{\mathrm{OC}}\right.$ of $0.71 \mathrm{~V}, \mathrm{~J}_{\mathrm{SC}}$ of $12.2 \mathrm{~mA} \mathrm{~cm}{ }^{-2}$, FF of 0.72), which was much higher than that for DSSCs with conventional ZTO-NPs-based photoelectrodes or those based on the popular ruthenium-based dye, N719. The unique morphology of the ZTO-Bs allowed for improvements in dye absorption, light scattering, electrolyte penetration, and the charge recombination lifetime, while the organic dyes resulted in high molar absorbability.

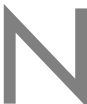
anocrystalline inorganic semiconductors have received considerable attention owing to their potential for use in Li-ion batteries, dye-sensitized solar cells (DSSCs), gas sensors, and photocatalysts for water splitting and organic pollutant degradation ${ }^{1-5}$. Although binary metal oxides (such as $\mathrm{TiO}_{2}, \mathrm{ZnO}$, and $\mathrm{SnO}_{2}$ ) are widely acknowledged as being highly suited semiconductor materials for these applications, ternary metal oxides such as $\mathrm{Zn}_{2} \mathrm{SnO}_{4}(\mathrm{ZTO})^{6}, \mathrm{Zn}_{2} \mathrm{TiO}_{3}^{7}$, and $\mathrm{BaSnO}_{3}$ have recently emerged as promising alternatives ${ }^{8}$. This is because ternary metal oxides exhibit several advantages. For instance, ternary metal oxides allow for a broader choice of materials. Further, they exhibit better corrosion resistance than do binary metal oxides. Finally, their chemical and electrical properties can be readily tailored by altering their composition. As a result, ternary metal oxides have recently been investigated for use as photoelectrode materials for DSSCs.

In particular, ZTO is being investigated extensively for use as a photoelectrode material for DSSCs because it exhibits a high electron mobility $\left(15 \mathrm{~cm}^{2} \mathrm{~V}^{-1} \mathrm{~s}^{-1}\right.$; in contrast that of $\mathrm{TiO}_{2}$ is $\left.0.1-1 \mathrm{~cm}^{2} \mathrm{~V}^{-1} \mathrm{~s}^{-1}\right)$ and an appropriate surface work function $(\sim 3.6 \mathrm{eV})$. In addition, it can also be readily synthesized in the form of nanoparticles'. Recently, Wu and coworkers reported the fabrication of DSSCs using ZTO nanoparticles (NPs) as the photoelectrode material. The DSCCs exhibited a power conversion efficiency (PCE) of $3.8 \%^{2}$. Similarly, our group fabricated amorphous ZTO nanofibers using the electrospinning method and used them as a photoelectrode material for DSSCs. The improved electron-transport properties of the ZTO nanofibers resulted in the cells exhibiting a PCE of 3.7\%. It is known that photoelectrode materials with a one-dimensional (1D) morphology have lower charge-recombination rates ${ }^{10}$; however, the increase in the performance of the cells owing to the use of the nanofibers was limited because charge transport in the amorphous ZTO nanofibers was not optimized ${ }^{10,11}$. Further improvements in ZTO nanomaterials in terms of their crystallinity and morphology, resulting in improved electron transport, dye absorption, and light scattering ${ }^{12,13}$, will lead to ZTO-based DSSCs showing even better performances. Further, the development of sensitizers that have high molar absorbabilities and are sufficiently compatibility with ZTO is also essential for improvements in the performance of ZTO-based DSSCs.

In this study, we fabricated high-efficiency DSSCs using novel ZTO materials and organic sensitizers. We first synthesized uniformly sized amphiphilic ZTO nanoparticles (ZTO-NP) in aqueous media. Next, we used a unique fabrication method to obtain hierarchically structured mesoporous ZTO beads (ZTO-B) through electrostatic spraying (e-spraying). Finally, we used organic sensitizers that exhibited high absorbabilities and were highly compatible with the ZTO beads to fabricate ZTO photoelectrodes. The submicron-scaled ZTO-Bs 

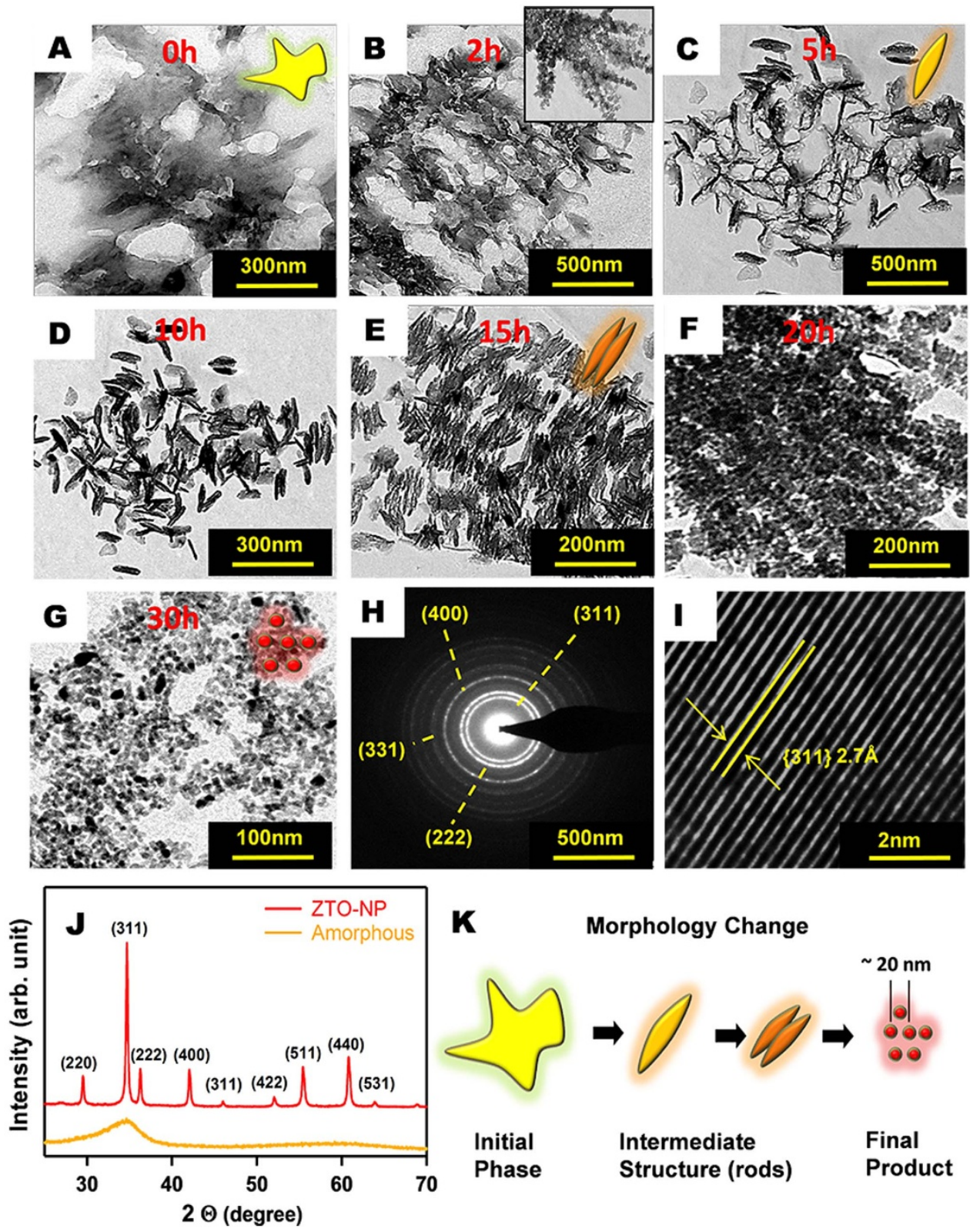

Morphology Change
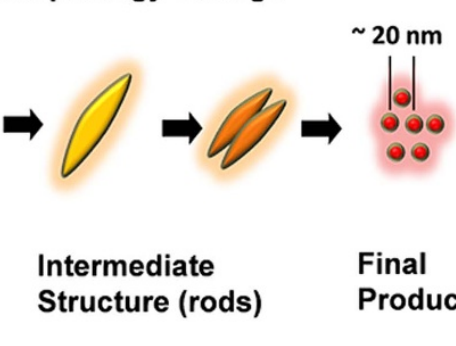

Final

Product

Figure 1 TEM images of the reaction products after various hydrothermal treatment times during ZTO synthesis $(A \sim$ F), ZTO-NPs after completion of the synthesis $(\mathrm{G})$, diffraction patterns of the polycrystalline ZTO nanoparticle powder $(\mathrm{H})$, high-resolution TEM images of the ZTO (I) (yellow lines illustrate ZTO fringes), XRD patterns ( $\mathrm{J}$ ), morphology changes during synthesis process $(\mathrm{K})$.

( $600 \mathrm{~nm}$ ), which contained embedded ZTO-NPs $(\sim 20 \mathrm{~nm})$, were fabricated by the e-spraying of a colloidal dispersion of the amphiphilic ZTO-NPs ${ }^{14-16}$. Two different organic sensitizers (SJ-T1 and SJET1), both of which have a donor- $\pi$-conjugated-acceptor (D- $\pi-A)$ structure, were synthesized and used as the dyes for the DSSCs. The performances of the organic dye-sensitized cells fabricated using the various ZTO-based photoelectrodes were investigated and compared with those of cells based on a conventional ruthenium complex dye (N719). Using the ZTO-B photoelectrodes led to the PCE of the corresponding DSSCs to be almost twice that of cells based on the ZTO-NP photoelectrodes. Further, the PCE values of the DSSCs based on the organic dyes were approximately thrice those of the cells based on the conventional N719 dye. The optimized SJ-ET1sensitized DSSCs displayed the highest PCE value, which was as high as $\sim 6.3 \%$, when 5 - $\mu$ m-thick ZTO-B photoelectrodes were used. To the best of our knowledge, this result is the highest value ever achieved in ternary metal oxide-based DSSCs.

\section{Results Section}

The amphiphilic ZTO-NPs were synthesized by the hydrothermal sol-gel method using $\mathrm{ZnSn}(\mathrm{OH})_{6}$ as the precursor. The fabrication method is described in the Experimental section and in Fig. S1 in Supporting Information (SI). The transmission electron microscopy (TEM) images in Fig. 1 show the evolution of the ZTO precursor, $\mathrm{ZnSn}(\mathrm{OH})_{6}$, into the ZTO-NPs through a sol-gel conversion process. The TEM images (Fig. 1A to G) were obtained after hydrothermal treatments of different durations $\left(\mathrm{T}_{\mathrm{D}}\right)$. In the early stage, small rods of $\mathrm{ZnSn}(\mathrm{OH})_{6}$, which had a size of $\sim 70 \mathrm{~nm}$ and whose surfaces were covered with crystalline ZTO, were formed (Fig.s 1A-F). The NPs started to form when $\mathrm{T}_{\mathrm{D}}$ was greater than $10 \mathrm{~h}$; these rods increased to approximately $10-30 \mathrm{~nm}$ in diameter after a reaction time of $30 \mathrm{~h}$ (Fig. 1G). The resulting ZTO-NPs were modified using an amphiphilic surfactant, tetrabutylammonium hydroxide (TBAOH), under ultrasonication, affording a stable colloidal dispersion of the ZTONPs in ethanol (Fig. S2). The zeta potential of the colloidal ZTO-NP 

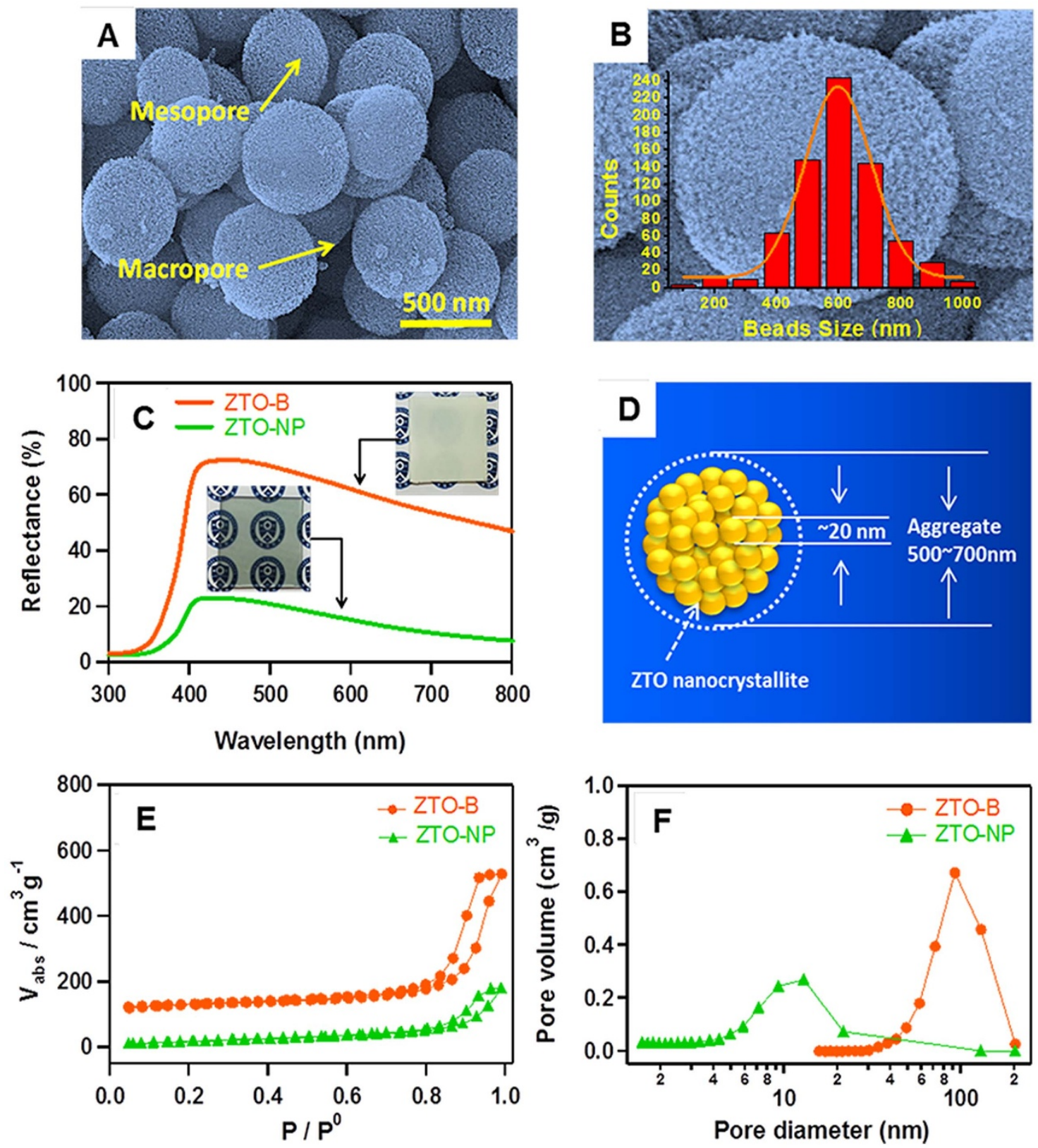

Figure 2 SEM images of the hierarchically structured e-sprayed ZTO-Bs on FTO/glass (A and B) and size distribution of ZTO-Bs used in this study (inserted graph - line is the fitted result by Gaussian function), illustration of ZTO-B (C), Diffused reflectance spectra (D), nitrogen sorption isotherms (E), and pore volume distribution of the ZTO based photoelectrodes (green: ZTO-NP, red: ZTO-B). The pore diameter was calculated using desorption

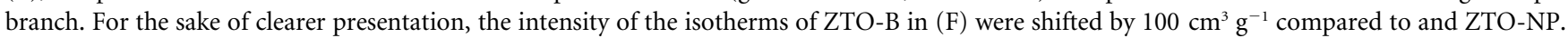

dispersion was less than $-35 \mathrm{mV}$, confirming that it was highly stable over a period of several months at room temperature. The average diameter of the ZTO-NPs was $\sim 20 \mathrm{~nm}$ (see TEM image in Fig. S1). The selected area electron diffraction (SAED) patterns (Fig. $1 \mathrm{H}$ ) of the NPs were indexed to the reflection of the polycrystalline ZTO in the structure. The X-ray diffraction (XRD) pattern of the ZTO-NPs is shown in Fig. 1J. The characteristic peaks of crystalline zinc stannate, that is, the (220), (311), (220), (400), (422) and (511) peaks, were observed after calcination. These peaks corresponded to a spinel structure, in which all the $\mathrm{Sn}^{4+}$ ions are octahedrally coordinated, while half of the $\mathrm{Zn}^{2+}$ ions are distributed in a tetrahedral coordination and the other half in an octahedral coordination. The estimated lattice parameter was $8.68 \AA$, which is in a good agreement with the reported value of $8.65 \AA$ (JCPDS Card No. 6-416). On the other hand, the XRD spectrum of the white precipitate formed after the mixing of the $\mathrm{Zn}^{2+}$ ions with the $\mathrm{Sn}^{2+}$ ions (the amorphous structure is shown in Fig. 1J, green line) did not correspond to that of ZTO. Thus, it can be concluded that the treatment of the ZTO precursor at $220^{\circ} \mathrm{C}$ for $30 \mathrm{~h}$ in the autoclave resulted in the formation of zinc stannate.

The ZTO-Bs were prepared by the e-spraying method using the ZTO colloidal solution (see Fig. S3 in SI). The colloidal dispersion of the ZTO-NPs was directly e-sprayed onto a fluorine-doped $\mathrm{SnO}_{2}$ (FTO)-coated glass substrate. The resulting submicron-scaled ZTO-Bs possessed a hierarchical structure (particles-in-beads), as can be seen from their scanning electron microscopy (SEM) images (Fig. 2A). The ZTO-Bs exhibited a relatively narrow size distribution, evenly covering the entire area of the substrate, as shown in Fig. $2 \mathrm{~B}$ and Fig. S3. The average diameter of the ZTO-Bs was determined to be $\sim 600 \mathrm{~nm}$ using Gaussian curve fitting (inset in Fig. 2B). It should be noted that the ZTO dispersion should exhibit sufficient colloidal stability and solvent polarity to allow for the fabrication of hierarch- 
Table 1 | The morphological properties of the ZTO photoelectrodes

\begin{tabular}{lccccc} 
ZTO & Surface area $\left(\mathrm{m}^{2} \mathrm{~g}^{-1}\right)$ & Pore volume $\left(\mathrm{cm}^{3} \mathrm{~g}^{-1}\right)$ & Porosity $(\%)^{[\mathrm{a}]}$ & Roughness Factor & Adsorbed Dye $\left.(\mathrm{molcm})^{-2}\right)^{[b]}$ \\
\hline ZTO-NP & 66.4 & 0.42 & 64.6 & 104.1 & $1.07 \times 10^{-8}$ \\
ZTO-B & 98.6 & 0.67 & 74.5 & 113.6 & $1.64 \times 10^{-8}$ \\
\hline
\end{tabular}

[a] The porosity were calculated by referring to the formula of $P=V p /\left(\rho^{-1}+V p\right)$, where $V p$ is the specific cumulative pore volume $\left(\mathrm{cm}^{3} \mathrm{~g}^{-1}\right)$ and $\rho^{-1}$ is the inverse of the density of $Z T O\left(\rho^{-1}=0.235 \mathrm{~cm}^{3} \mathrm{~g}^{-1}\right)$. [b] The SJ-ET1 was used for adsorbed dye analysis.

ically structured ZTO-Bs by the e-spraying process ${ }^{15}$. The size of the ZTO-Bs could be controlled by manipulating the e-spraying parameters such as the applied voltage, the concentration of ZTO in the dispersion, and the solvent composition. In this study, we chose to fabricate ZTO-Bs with an average size of $\sim 600 \mathrm{~nm}$ in order to enhance the degree of visible light-scattering and hence the lightharvesting efficiency of the cells (Fig. 2C) ${ }^{16}$. Within the ZTO-Bs, the embedded primary ZTO-NPs were closely packed together, with the ZTO-Bs exhibiting the particles-in-beads structure shown in Fig. 2D.

The morphological characteristics of the ZTO-Bs, such as their specific surface area, porosity, and surface roughness, were determined using the Brunauer-Emmett-Teller (BET) method (Fig. 2E and F). The results are listed in Table 1 . In order to elucidate the effects of the morphological characteristics of the fabricated ZTO-Bs on their performance as a photoelectrode material for DSSCs, we also prepared ZTO-NP-based photoelectrodes by e-spraying a colloidal solution of ZTO-NPs that did not exhibit a "particles-in-beads" structure and compared the performances of the two. The specific surface areas of the e-sprayed ZTO-Bs and ZTO-NPs were $98.6 \mathrm{~m}^{2}$ $\mathrm{g}^{-1}$ and $66.4 \mathrm{~m}^{2} \mathrm{~g}^{-1}$, while the porosities (P) of the ZTO-Bs and ZTONPs were $74.5 \%$ and $64.6 \%$, respectively (Table 1 ). These results demonstrated that the ZTO-B-based photoelectrodes had a larger surface area and higher porosity than did the ZTO-NP-based photoelectrodes. The estimated roughness values $(R)$ per unit thickness of the photoelectrodes were determined using the following formula: $R$ $=\rho(1-P) S$, where $\rho$ is the density $\left(\mathrm{g} \mathrm{cm}^{-3}\right)$ of the ZTO nanomaterial, $P$ is the porosity (\%) of the ZTO film, and $S$ is the specific surface area $\left(\mathrm{m}^{2} \mathrm{~g}^{-1}\right)$. The R values of the ZTO-B and ZTO-NP photoelectrodes were 113.6 and 104.1, respectively. The increased surface area of the ZTO-Bs resulted in them exhibiting a dye-loading capacity $\left(16.4 \mathrm{nmol} \mathrm{cm} \mathrm{cm}^{-2}\right)$ that was more than $50 \%$ higher than that of the ZTO-NPs $\left(10.7 \mathrm{nmol} \mathrm{cm}^{-2}\right)$, as shown in Table 1 and Fig. S4.

Next, we synthesized the organic dyes used, which had a structure in which a long alkoxy group-substituted triphenylamine (TPA) donor moiety was connected to a cyanoacetic acid acceptor moiety through conjugation with either a thiophene (T) or an 3,4-ethylenedioxythiophene (EDOT) group. We chose the ethylhexyloxy substituent on TPA, because the bulky alkoxy group is known to prevent charge recombination between the photoelectrode material and the redox couples (usually $\mathrm{I}^{-} / \mathrm{I}_{3}{ }^{-}$) in the electrolyte, in addition to exhibiting electron-donor-like characteristics ${ }^{17,18}$. The structure of the organic sensitizers SJ-T1 and SJ-ET1 are shown in Fig. 3A, and the process for synthesizing them is depicted in Fig. S5. The molar absorbabilities of the dyes at their maximum absorption wavelengths were $48,000 \mathrm{M}^{-1} \mathrm{~cm}^{-1}$ for SJ-T1 (at $488 \mathrm{~nm}$ ) and 42,500 $\mathrm{M}^{-1} \mathrm{~cm}^{-1}$ for SJ-ET1 (at $495 \mathrm{~nm}$ ); these values are much higher than that for the popular ruthenium dye $\operatorname{N7} 19\left(\varepsilon=13000 \mathrm{M}^{-1} \mathrm{~cm}^{-1} \text { at } 535 \mathrm{~nm}\right)^{2}$. The optical band gaps of SJ-T1 and SJ-ET1, determined from their ultraviolet-visible (UV-vis) spectra, were $1.96 \mathrm{eV}$ and $1.93 \mathrm{eV}$, respectively (Fig. 3B).

DSSCs were fabricated using the ZTO-based photoelectrodes (DSSC-ZTOs) and the D- $\pi$-A organic sensitizers. Fig. 4A shows the $J-V$ characteristics of the DSSC-ZTOs with SJ-ET1 as the sensitizer; the characteristics were measured under AM 1.5 G one sun illumination. The photoelectrochemical parameters determined from the $J-V$ characteristics are summarized in Table 2. The PCE of the SJ-ET1-sensitized DSSC-ZTO-B was found to be $6.2 \%$, which was approximately two times higher than that of the DSSC-ZTONPs (3.5\%); the former device also exhibited improved open-circuit voltage $\left(V_{\mathrm{OC}}\right)$, short-circuit current density $\left(J_{\mathrm{SC}}\right)$, and fill factor $(\mathrm{FF})$ values. This value is also much higher than those obtained in other studies on ZTO-based DSSCs (3.5-4.7\%) $)^{13,19-20}$, including those reported by our group $(3.7 \%)^{10}$. To allow for a better comparison, we also fabricated DSSCs based on ZTO-NP photoelectrodes prepared using the conventional doctor's blade method (Fig. S10). We found that the ZTO-NP-based DSSCs fabricated using the two techniques exhibited similar performances (3.5\% vs. 3.3\%); these values were much lower than that for the DSSC-ZTO-Bs. The maximum external quantum efficiency (EQE) value of the DSSC-ZTO-Bs at $480 \mathrm{~nm}$ was $74 \%$, whereas that of the DSSC-ZTO-NPs was $56 \%$ (Fig. 4B). That the $J_{\mathrm{SC}}$ and EQE of the DSSC-ZTO-Bs were higher can be attributed to their improved light-harvesting capability. Further, the greater specific surface area and roughness factor of the ZTO-Bs resulted in them exhibiting a dye adsorption rate that was $\sim 53 \%$ higher than that for ZTO-NPs (Table 1). The improvement in light scattering in the case of the submicron-sized ZTO-Bs resulted in improved light harvesting in the visible region, as indicated by the reflectance data shown in Fig. 2C.

The $J-V$ characteristics of the DSSC-ZTOs based on the different dyes and photoelectrodes are shown in Fig.s S8 and S9 and Table S4 and S5 in SI. Regardless of the type of ZTO photoelectrode used, the organic dye-based DSSCs displayed better photoconversion characteristics than those of the N719-based DSSCs (Fig. S8 and Table S4). That the molar extinction coefficients of the organic dyes (SJ-T1 and SJ-ET1) were higher than those of N719, the most commonly used ruthenium-based dye, can be deduced from the fact that the shortcircuit current density of the cells based on the former was higher. The high absorbability of the organic dyes also allowed for the required thickness of the ZTO photoelectrodes to be reduced, lowering the charge-transport distance. The highest $J_{\mathrm{SC}}$ value of the organic dye-based DSSC-ZTOs was exhibited at a thickness of $\sim 5 \mu \mathrm{m}$; at this thickness, the absorption level and the rate of charge transport were at the optimal levels. On the other hand, the optimal photoelectrode thickness was $>10 \mu \mathrm{m}$ in the case of the N719-based DSSCs (Fig.s S8 and S9, Tables S4 and S5).

In addition to improving light harvesting, the hierarchical morphology of the ZTO-Bs also enhanced the charge-transport properties in the resultant DSSCs. The internal series resistance $\left(R_{\mathrm{S}}\right)$ values of the cells, which are closely related to their FF values, were determined through electrochemical impedance (EIS) analysis. The procedure for determining the $R_{\mathrm{S}}$ values of the DSSCs from the results of the EIS-based characterization of the DSSC-ZTOs is shown in Fig.s S11 and S12. The Nyquist plot in Fig. S11A shows that the $R_{\mathrm{S}}$ values of the DSSC-ZTO-Bs were lower than that of the DSSC-ZTONPs, regardless of the dye used. This result was in a good agreement with the fact that the FF of the DSSC-ZTO-Bs was higher than that of the DSSC-ZTO-NPs (Table S6). This can be attributed to the presence of macropores in ZTO-Bs, which facilitated the penetration of the electrolyte into the ZTO photoelectrodes, allowing the surfaces of the photoelectrodes to be wetted to a greater degree. This phenomenon has been noticed in photoelectrodes based on other hierarchical materials as well ${ }^{14,15}$.

The charge-transport times $\left(\tau_{\mathrm{CT}}\right)$ and recombination lifetimes $\left(\tau_{\mathrm{R}}\right)$ of the DSSC-ZTOs were determined using the intensity-modu- 

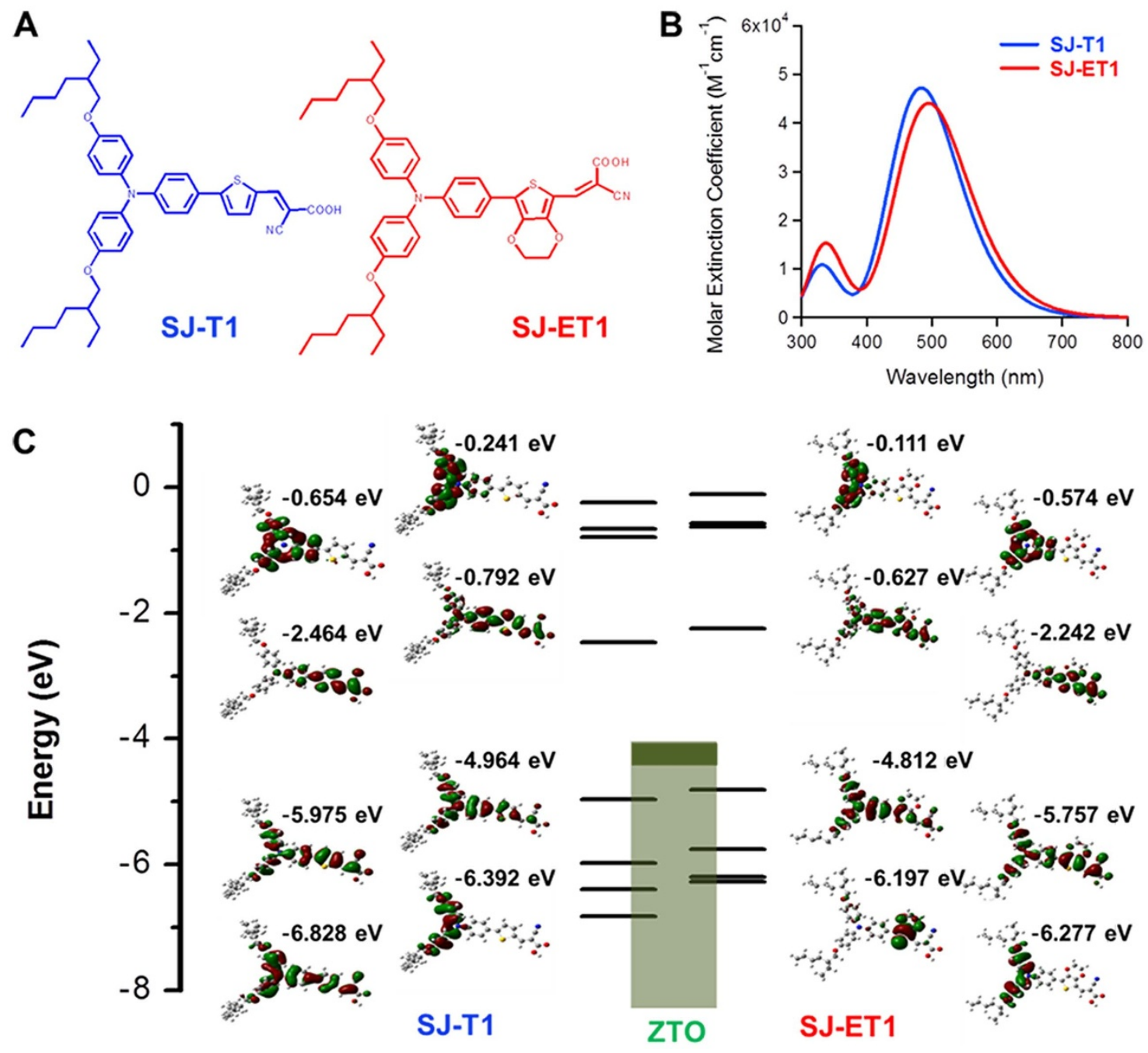

Figure 3 The chemical structures of the organic sensitizers (A), absorption spectrum (B), and Energetic MO levels and Frontier MOs of SJ-T1 and SJET1 (C).

lated photocurrent/photovoltage (IMPS/IMVS) analysis techniques; the results are shown in Fig.s $4 \mathrm{C}-\mathrm{F}$. The $\tau_{\mathrm{CT}}$ values were estimated from the results of the IMPS analysis using the following relation: $\tau_{\mathrm{CT}}$ $=1 / 2 \pi f_{\min (\mathrm{IMPS})}$, where $f_{\min }$ is the minimum current of the imaginary part of the low-frequency range in the IMPS spectra (Fig. S13). The $\tau_{\mathrm{CT}}$ value of the DSSC-ZTO-Bs was more than two times smaller than that of the DSSC-ZTO-NPs; this can be attributed to the lower junction density of the hierarchical structure of the ZTO-Bs. The tightly connected primary ZTO-NPs packed within the ZTO-Bs (see the SEM images in Fig. 2B) exhibited a lower resistance for charge transport compared to the randomly stacked ZTO-NPs; this was regardless of whether they were fabricated by e-spraying or using a doctor's blade. The net charge transport was greater in the ZTO-B photoelectrodes than in the ZTO-NP photoelectrodes, even though the total charge-transport distance may have been greater in the former owing to the pore volume being higher. The $\tau_{R}$ values were estimated from the results of the IMVS analysis (Fig. 4D) using the following relation: $\tau_{r}=1 / 2 \pi f_{\min (\mathrm{IMVS})}$, where $f_{\min }$ is the minimum voltage in the imaginary part of the low-frequency range of the IMVS spectra. The $\tau_{\mathrm{R}}$ value of the DSSC-ZTO-Bs was $\sim 6$ times higher than that of the DSSC-ZTO-NPs under an incident photon flux of $3.18 \times$ $10^{18} \mathrm{~cm}^{-2} \mathrm{~s}^{-1}$, as shown in Fig. S13B in SI. The electron-diffusion length $\left(D_{\mathrm{L}}\right)$ of the DSSC-ZTO-Bs, calculated using the equation $D_{\mathrm{L}}=$ $\left(D \times \tau_{\mathrm{R}}\right)^{1 / 2}$, where $D$ is the diffusion coefficient obtained from the IMPS analysis, was $\sim 25 \mu \mathrm{m}$ while that of the DSSC-ZTO-NPs was only $\sim 6 \mu \mathrm{m}$ (Fig. $4 \mathrm{~F}$ ). This result indicated that the probability of the electrons arriving from the ZTO-B photoelectrode layers to the charge-collecting electrode (FTO) was considerably higher than that of them arriving from the ZTO-NP photoelectrodes. The chargecollection efficiency $\left(\eta_{\mathrm{CC}}\right)$ of the DSSC-ZTO-Bs, determined from the relationship $\eta_{\mathrm{CC}}=1-\left(\tau_{\mathrm{CT}} / \tau_{\mathrm{R}}\right)$, was an order of magnitude greater than that of the DSSC-ZTO-NPs, owing to the longer charge-recombination lifetime and shorter electron-transport time of the former. The higher $\eta_{\mathrm{CC}}$ coupled with the higher dye adsorption resulted in an increase in the $J_{\mathrm{SC}}$ and FF values of the DSSC-ZTO-Bs (Table 2).

The photoelectron density (PED), $n$, in a ZTO photoelectrode determines its quasi-Fermi level, which, in turn, determines the $V_{\mathrm{OC}}$ value of the corresponding DSSC. Generally, the PED is strongly correlated to the $\tau_{\mathrm{R}}$ value as per Equation $(1)^{21}$ :

$$
n=\frac{q \varphi I_{0} \tau R}{q(1-p) d}
$$

where $q$ is the charge of an electron, $\varphi$ is the ratio of injected electrons to incident photons, $I_{0}$ is the incident photon flux density, $p$ is the film porosity, and $d$ is the film thickness. Therefore, the PED of the DSSC-ZTO-Bs was estimated to be more than six times higher than that of the DSSC-ZTO-NPs, resulting in an increase in the $V_{\mathrm{OC}}$ value to up to $70 \mathrm{mV}$. That the $V_{\mathrm{OC}}$ of the DSSC-ZTO-Bs was higher could also be confirmed from the EIS results. The maximum frequency $\left(f_{\max }\right)$ in the middle range of the Bode phase plots (Fig. S11B) for 

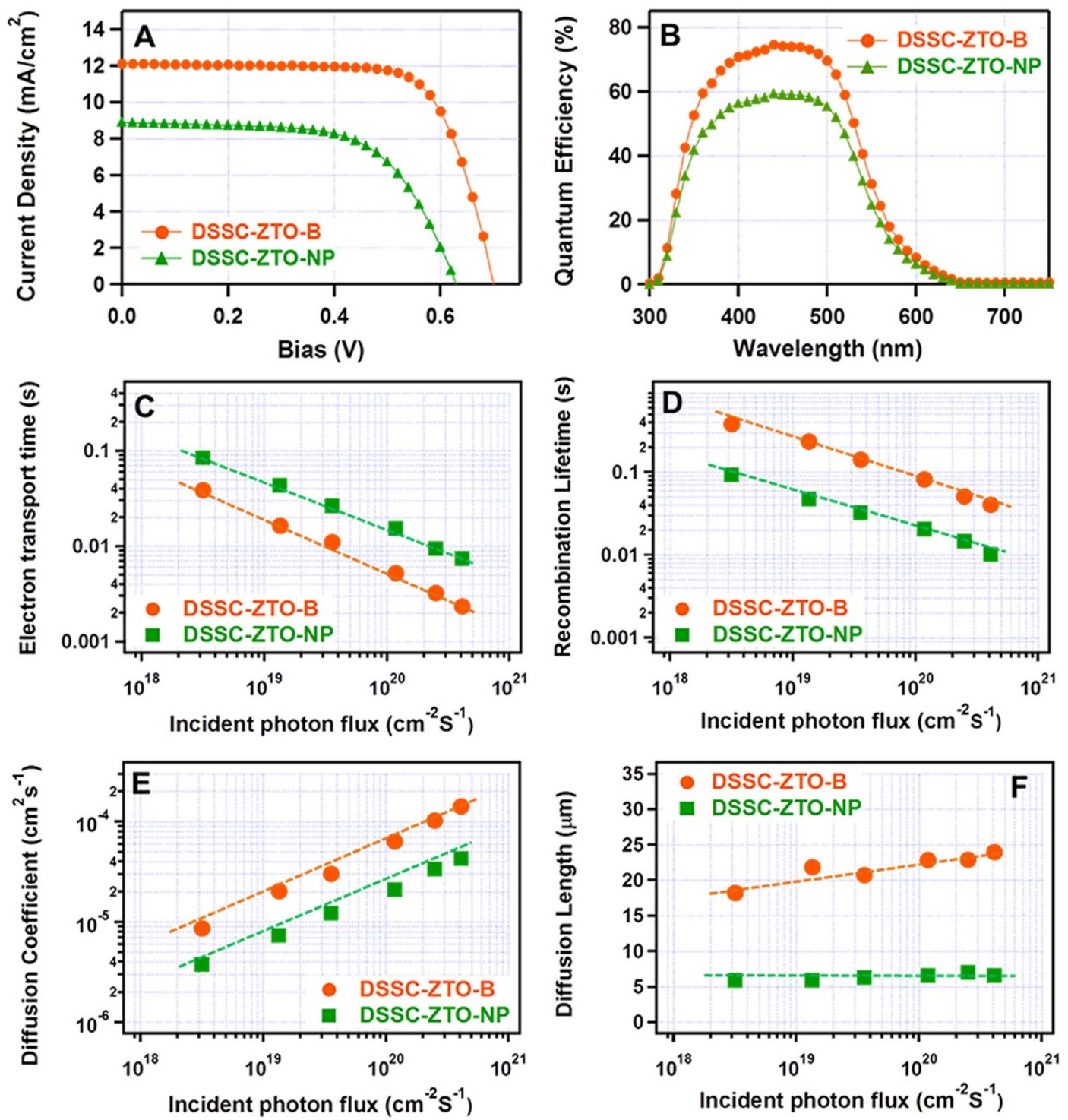

Figure $4 \mid J$ - $V$ characteristics (A) and incident-photon-to-current conversion efficiency (IPCE) spectra (B) of the DSSC-ZTOs using various photoelectrodes under simulated AM $1.5 \mathrm{G}$ solar radiation at $100 \mathrm{mWcm}^{-2}$ with SJ-ET1 sensitizer. Analysis results of intensity modulated photoelectron measurements of DSSC-ZTOs with respect to the incident photon flux; plots of electron transport time $\left(\tau_{\mathrm{CT}}\right)(\mathrm{C})$, diffusion coefficient $(\mathrm{D})$, charge recombination lifetime $\left(\tau_{\mathrm{R}}\right)(\mathrm{E})$, and charge diffusion length $\left(\mathrm{D}_{\mathrm{L}}\right)(\mathrm{F})$. The frequency of incident light modulation ranged from 100 to $0.1 \mathrm{~Hz}$, and SJ-ET1 was used as the sensitizer.

the DSSC-ZTO-Bs was much lower than that for the DSSC-ZTONPs. Under illumination, the relationship between $V_{\mathrm{OC}}$ and the recombination lifetime can be described by Equation $(2)^{22}$ :

$$
V_{o c}=\frac{R T}{\beta F} \ln \left[\frac{A I}{n_{0} k_{1}\left[I_{3}^{-}\right]+n_{0} k_{2}\left[D^{+}\right]}\right]
$$

where $R$ is the molar gas constant, $T$ is the temperature, $F$ is the Faraday constant, $\beta$ is the reaction order for $\mathrm{I}_{3}$ and electrons, $A$ is the electrode area, $I$ is the incident photon flux, $n_{0}$ is the concentration of accessible electronic states in the conduction band, and $k_{1}$ and $k_{2}$ are the kinetic constants of the back reaction of the injected electrons with $\mathrm{I}_{3}{ }^{-}$and the recombination of these electrons with oxidized dyes, respectively. Assuming that recombination with the oxidized dye molecules can be neglected, $V_{\mathrm{OC}}$ depends logarithmically on the kinetic constant of the back reaction of the injected electrons with $\mathrm{I}_{3}$ $\left(k_{1}\right)$. As the concentration of $\mathrm{I}_{3}{ }^{-}$in the electrolyte and the incident photon flux were constant under the experimental conditions, $V_{\mathrm{OC}}$ was proportional to $\ln \left(1 / f_{\max }\right)$, demonstrating that the charge- recombination rate influenced the $V_{\mathrm{OC}}$ value of the DSSCs significantly. From this relationship, the $V_{\mathrm{OC}}$ value of the DSSC-ZTO-Bs was found to be $\sim 70 \mathrm{mV}$ higher than that of the DSSC-ZTO-NPs. The consistency in the $V_{\mathrm{OC}}$ shifts estimated from the results of the IMVS analysis and EIS confirmed further that the fabricated ZTO-Bs exhibited a lower charge-recombination rate compared to than in the ZTO-NPs.

\begin{tabular}{|c|c|c|c|c|c|}
\hline Photo-electrodes & Dye & $V_{O C}(V)$ & $J_{\mathrm{SC}}\left(\mathrm{mAcm}{ }^{-2}\right)$ & $\mathrm{FF}$ & PCE (\%) \\
\hline \multirow[t]{3}{*}{ ZTO-B } & SJ-T1 & 0.69 & 11.3 & 0.69 & 5.5 \\
\hline & SJ-ET 1 & 0.71 & 12.2 & 0.72 & 6.3 \\
\hline & N719 & 0.72 & 6.4 & 0.65 & 3.0 \\
\hline \multirow[t]{3}{*}{ ZTO-NP } & SJ-TI & 0.65 & 7.5 & 0.62 & 3.0 \\
\hline & SJ-ET 1 & 0.64 & 8.6 & 0.64 & 3.5 \\
\hline & N719 & 0.71 & 5.3 & 0.61 & 2.3 \\
\hline
\end{tabular}




\section{Discussion Section}

We developed a novel strategy for synthesizing uniformly sized amphiphilic ZTO-NPs in aqueous media, as well as hierarchically structured ZTO-Bs, by the e-spraying method using a colloidal dispersion. Highly absorbable organic dyes (SJ-T1 and SJ-ET1) were synthesized and used as the sensitizers. To the best of our knowledge, the DSSCs based on the fabricated ZTO-Bs and organic dyes exhibited the highest performance yet reported for DSSCs fabricated using ternary metal oxide-based photoelectrodes. The optimized DSSCZTO-Bs based on SJ-ET1 showed a PCE of $6.25 \%$ ( $V_{\text {OC }}$ of $0.71 \mathrm{~V}$, $J_{\mathrm{SC}}$ of $12.2 \mathrm{~mA} \mathrm{~cm}^{-2}, \mathrm{FF}$ of 0.72 ), which was approximately two times higher than that of the DSSC-ZTO-NPs (PCE of $3.5 \%$ with $V_{\mathrm{OC}}$ of $0.64 \mathrm{~V}, J_{\mathrm{SC}}$ of $8.6 \mathrm{~mA} \mathrm{~cm}^{-2}$, FF of 0.64 ) as well as that of other devices reported in the literature. The high molar absorbability of the organic dyes fabricated in this study as well as the hierarchical morphology of the ZTO-Bs resulted in the drastic increase in performance. The unique morphology of the ZTO-Bs, in which the high-crystallinity primary ZTO-NPs were embedded within submicron-scaled beads, led to improved dye absorption, light scattering, and electrolyte penetration and a lower charge-recombination lifetime. These improvements, in turn, resulted in enhancements in the photoelectron-conversion properties of the devices. Considering that the improved performances were obtained using films of relatively low thicknesses $(<5 \mu \mathrm{m})$, the results suggest that the proposed fabrication technique should be suited for fabricating solid-state and/or flexible DSSCs as well.

1. Yan, N. et al. Hollow Porous $\mathrm{SiO}_{2}$ Nanocubes Towards High-performance Anodes for Lithium-ion Batteries. Sci. Rep. 3, 1568, DOI: 10.1038/srep01568 (2013).

2. O'Regan, B. \& Gratzel, M. A low-cost, high-efficiency solar cell based on dyesensitized colloidal $\mathrm{TiO}_{2}$ films. Nature 353, 737-740 (1991).

3. Gong, J., Li, Y., Hu, Z., Zhou, Z. \& Deng, Y. Ultrasensitive $\mathrm{NH}_{3}$ Gas Sensor from Polyaniline Nanograin Enchased $\mathrm{TiO}_{2}$ Fibers. J. Phys. Chem. C 114, 9970-9974 (2010).

4. Kim, I.-D. et al. Ultrasensitive Chemiresistors Based on Electrospun $\mathrm{TiO}_{2}$ Nanofibers. Nano Lett. 6, 2009-2013 (2006).

5. Kong, M. et al. Tuning the Relative Concentration Ratio of Bulk Defects to Surface Defects in $\mathrm{TiO}_{2}$ Nanocrystals Leads to High Photocatalytic Efficiency. J. Am. Chem. Soc. 133, 16414-16417 (2011).

6. Tan, B., Toman, E., Li, Y. \& Wu, Y. Zinc Stannate $\left(\mathrm{Zn}_{2} \mathrm{SnO}_{4}\right)$ Dye-Sensitized Solar Cells. J. Am. Chem. Soc. 129, 4162-4163 (2007).

7. Bae, C. et al. Template-Directed Synthesis of Oxide Nanotubes: Fabrication, Characterization, and Applications †. Chem. Mater. 20, 756-767 (2008).

8. Shin, S. S. et al. Improved Quantum Efficiency of Highly Efficient Perovskite $\mathrm{BaSnO}_{3}$-Based Dye-Sensitized Solar Cells. ACS Nano 7, 1027-1035 (2013).

9. Coutts, T. J., Young, D. L., Li, X., Mulligan, W. P. \& Wu, X. Search for improved transparent conducting oxides: A fundamental investigation of $\mathrm{CdO}, \mathrm{Cd}_{2} \mathrm{SnO}_{4}$, and $\mathrm{Zn}_{2} \mathrm{SnO}_{4}$. J. Vac. Sci. Technol. A 18, 2646-2660 (2000).

10. Choi, S.-H. et al. Amorphous Zinc Stannate $\left(\mathrm{Zn}_{2} \mathrm{SnO}_{4}\right)$ Nanofibers Networks as Photoelectrodes for Organic Dye-Sensitized Solar Cells. Adv. Func. Mater. 23, 3146-3155 (2013).

11. Rajachidambaram, J. S. et al. Characterization of amorphous zinc tin oxide semiconductors. J. Mater. Res. 27, 2309-2317 (2012).

12. Liu, R. et al. Fabrication of $\mathrm{Zn}_{2} \mathrm{SnO}_{4} / \mathrm{SnO}_{2}$ hollow spheres and their application in dye-sensitized solar cells. RSC Adv. 3, 2893-2896 (2013).
13. Chen, J., Lu, L. \& Wang, W. $\mathrm{Zn}_{2} \mathrm{SnO}_{4}$ Nanowires as Photoanode for DyeSensitized Solar Cells and the Improvement on Open-Circuit Voltage. J. Phys. Chem. C 116, 10841-10847 (2012).

14. Hwang, D. et al. Electrospray Preparation of Hierarchically-structured Mesoporous $\mathrm{TiO}_{2}$ Spheres for Use in Highly Efficient Dye-Sensitized Solar Cells. ACS Appl. Mater. Interfaces 3, 2719-2725 (2011).

15. Hwang, D., Kim, D. Y., Jang, S.-Y. \& Kim, D. Superior photoelectrodes for solidstate dye-sensitized solar cells using amphiphilic $\mathrm{TiO}_{2}$. J. Mater. Chem. A 1, 1228-1238 (2013).

16. Hulst, H. C. v. d. Light Scattering by Small Particles. [63-101] (Wiley, New York, 1957).

17. Li, R., Liu, J., Cai, N., Zhang, M. \& Wang, P. Synchronously Reduced Surface States, Charge Recombination, and Light Absorption Length for HighPerformance Organic Dye-Sensitized Solar Cells. J. Phys. Chem. B 114, 4461-4464 (2010).

18. Zhang, M. et al. Design of high-efficiency organic dyes for titania solar cells based on the chromophoric core of cyclopentadithiophene-benzothiadiazole. Energy Environ. Sci. 6, 2944-2949 (2013).

19. Kim, D. W. et al. Synthesis and photovoltaic property of fine and uniform $\mathrm{Zn}_{2} \mathrm{SnO}_{4}$ nanoparticles. Nanoscale 4, 557-562 (2012).

20. Li, Y. et al. Incorporating $\mathrm{Zn}_{2} \mathrm{SnO}_{4}$ Quantum Dots and Aggregates for Enhanced Performance in Dye-Sensitized ZnO Solar Cells. Chem. Eur. J. 18, 11716-11722 (2012).

21. Adachi, M., Sakamoto, M., Jiu, J., Ogata, Y. \& Isoda, S. Determination of Parameters of Electron Transport in Dye-Sensitized Solar Cells Using Electrochemical Impedance Spectroscopy. J. Phys. Chem. B 110, 13872-13880 (2006).

22. Wang, Q., Moser, J.-E. \& Gratzel, M. Electrochemical Impedance Spectroscopic Analysis of Dye-Sensitized Solar Cells. J. Phys. Chem. B 109, 14945-14953 (2005).

\section{Acknowledgments}

The authors gratefully acknowledge support from the Basic Science Research Program of the National Research Foundation (NRF) of Korea (2012045675); the New \& Renewable Energy Core Technology Program of the Korea Institute of Energy Technology Evaluation and Planning (KETEP), which is funded by the Ministry of Trade, Industry \& Energy, Republic of Korea (No. 20133030000210) (S.Y.J.); the Global Frontier R\&D Program of the Center for Multiscale Energy System, funded by the NRF through the Ministry of Education, Science and Technology, Korea (2012-8-2018) (D.K.); and the KIST Institutional Programs (2E23900 and 2K02120) (D.Y.K.).

\section{Author contributions}

D.H., H.L., D.K. and S.Y.J. wrote the main manuscript text and Fig. 1 and 2. J.-S.J. and H.C. prepared Fig. 3. D.Y.K. and H.-J.K. prepared Fig. 4 and wrote parts of discussion section. All authors reviewed the manuscript.

\section{Additional information}

Supplementary information accompanies this paper at http://www.nature.com/ scientificreports

Competing financial interests: The authors declare no competing financial interests.

How to cite this article: Hwang, D. et al. Hierarchically Structured $\mathrm{Zn}_{2} \mathrm{SnO}_{4}$ Nanobeads for High-Efficiency Dye-Sensitized Solar Cells. Sci. Rep. 4, 7353; DOI:10.1038/srep07353 (2014).

This work is licensed under a Creative Commons Attribution-NonCommercialNoDerivs 4.0 International License. The images or other third party material in this article are included in the article's Creative Commons license, unless indicated otherwise in the credit line; if the material is not included under the Creative Commons license, users will need to obtain permission from the license holder in order to reproduce the material. To view a copy of this license, visit http:// creativecommons.org/licenses/by-nc-nd/4.0/ 